\title{
Buenas Prácticas Agrícolas. Aproximaciones a una ruptura con las relaciones tradicionales entre productores frutícolas vinculados a la organización Fundación Horizonte Verde ${ }^{1}$
}

\author{
Autores: Rosario Joaquín Reales Vega² \\ Correspondencia en: rosario.joaquin@curnvirtual.edu.co \\ Cvlac \\ http://scienti1.colciencias.gov.co:8081/cvlac/visualizador/generarCurriculoCv.do?cod_rh=00000320
}

18

\section{RESUMEN}

En este documento se exponen algunas consideraciones relacionadas con el inicio de lo que aquí se puede denominar el establecimiento de Buenas Prácticas Agrícolas en interior de una organización sin ánimo de lucro ubicada en el corregimiento de Santa Cruz, municipio de Luruaco, al norte de Colombia, que han iniciado el cambio de una mentalidad agrícola anclada y practicada desde lo tradicional. En forma general se ha planteado para el proyecto el siguiente objetivo: Interpretar la forma como asimilan los productores frutícolas adscritos organizados, la aplicación de buenas prácticas agrícolas y de relaciones socio/jurídicas. La metodología empleada para la recolección de información, para el análisis e interpretación de la misma se fundamentó en el paradigma de investigación socio-crítico con la modalidad de Investigación-Acción-Participación. Entre los principales resultados de la investigación se encuentran: El inicio del cambio de actitud con el uso de Buenas Prácticas Agrícolas y la puesta en escena de hábitos jurídicos hacia una cultura de lo legal.

\section{Palabras clave}

Fortalecimiento, Relaciones familiares, Relaciones jurídicas, Buenas Prácticas Agrícolas, Productores frutícolas, Organización.

\begin{abstract}
In this document some considerations related to the beginning are exposed of what here can be named the establishment of Good Agricultural Practices in interior of a non-profit-making

\footnotetext{
${ }^{1}$ Este Artículo responde a los primeros avances del estudio Fortalecimiento de las relaciones familiares y jurídicas en la aplicación de Buenas Prácticas Agrícolas entre productores frutícolas en el Departamento del Atlántico vinculados a la organización Fundación Horizonte Verde. de la Corporación Universitaria Rafael Núñez Sede Barranquilla
}

${ }^{2}$ Sociólogo. Especialista en Gestión de Proyectos Educativos. Adscrito al Grupo Andrés Bello del Semillero de Investigación
\end{abstract}


Vol. 6, No. 1 Diciembre de 2014 pp. $121-135$

organization located in the corregimiento of Santa Cruz, Luruaco's municipality, to the north of Colombia, which they have initiated the change of an agricultural mentality anchored and practised from the traditional thing. In general form the following aim has appeared for the project: To interpret the form since there assimilate the fruit assigned organized producers, the application of good agricultural practices and of relations socio/juridical. The methodology used for the compilation of information, for the analysis and interpretation of the same one partner - critic was based on the paradigm of investigation.

\section{Key Word}

Strengthening; familiar Relations; juridical Relations; Good Agricultural Practices; fruit Producers; Organization.

\section{INTRODUCCIÓN}

Las Primeras manifestaciones de cambio manifestadas por los integrantes del Proyecto Fortalecimiento de las relaciones familiares y jurídicas en la aplicación de Buenas Prácticas Agrícolas entre productores frutícolas en el Departamento del Atlántico vinculados a la organización Fundación Horizonte Verde fue su cambio de actitud con respecto a la utilización de Buenas Prácticas Agrícolas, estaba en su vocabulario cuando hacían referencia a la utilización de buenos hábitos para las labores agrícolas. También este cambio se percibió entre algunos funcionarios que conformas la Junta Directiva de la Organización que les sirve de soporte en la ejecución del proyecto: La fundación Horizonte Verde Horve-.

No se puede decir que este sea un cambio definitivo; tampoco se puede asegurar que de ahora en adelante la situación en cuanto al desarrollo de actividades y la ejecución de las mismas será en un altísimo porcentaje al máximo. Lo que sí se puede asegurar es que tendrán encuentros con situaciones difíciles que le plantearán retos, tanto a los Miembros de Junta Directiva como a los asociados y/o beneficiarios de los proyectos. En este recorrido por la organización se pudieron encontrar diferentes circunstancias que evidencian cierta transformación, una especie de ruptura de la forma como se venían estableciéndose las relaciones entre productores frutícolas vinculados a la entidad organización Fundación Horizonte Verde. 
Con eventos de observación participante en los Talleres de capacitación a los productores de mango y en las jornadas de adiestramiento con cada uno de los componentes del Proyecto (ambiental, agroindustrial y empresarial), se han abordado los primeros elementos que denotan el comienzo de una ruptura.

\section{MATERIALES Y METODO}

Se ha implementado como procedimiento metodológico en el proceso investigativo, en la recolección de información, los fundamentos de la Investigación-Acción- Participación IAP (Fals Borda, O., 1998), basada en la etnografía. Por ser de carácter cualitativo en la información que se recolecte, se utilizarán técnicas como la entrevista etnográfica, la observación etnográfica, los grupos focales, la memoria de ancianos, en la construcción de memoria campesina (Molano Camargo, F., 2012). La estructura metodológica ha sido definida con la implementación de técnicas propias de los procesos investigativos cualitativos orientados por la Investigación-Acción Participación IAP. En tal sentido y coherente con el método etnográfico se tiene para la recolección de la información las siguientes técnicas: Observación etnográfica (participante); Entrevista etnográfica con los beneficiaros del proyecto y los miembros de su familia mayores de edad o que asistan al colegio; Recuperación bibliográfica de las más recientes publicaciones sobre el tema; Grupos focales (reunión de un grupo - entre 6 y 12 personas); Memoria de ancianos.

Por ser información cualitativa estos datos serán procesados mediante el uso de programas específicos para tal fin entre los que se resaltan: NUD*IST6, NUD*IST vivo 2, ATLAS ti, AQUAD 5, Etnograph 5, WINMAX, MAXQDA, los cuales se utilizarán en el procesamiento, organización e integración, representación grafica, el trabajo en equipo de la información recolectada. En el análisis de la información se recurrirá a la triangulación de datos, procedimiento que consiste en el cruce de información proveniente de las diferentes fuentes. 


\section{DESARROLLO. DISCUSIÓN}

\section{Estado del arte de la investigación}

El escenario natural del proyecto, es decir la ubicación geográfica corresponde al corregimiento de Santa Cruz, municipio de Luruaco, Departamento del Atlántico, al norte de la República de Colombia.

Los proyectos productivos que en la actualidad se desarrollan en el país se organizan mediante la figura de Alianzas Productivas, una modalidad de ejecución financiera que compromete a los entes territoriales y a las organizaciones comunitarias orientadas al trabajo mediante un determinado proyecto agropecuario. En el país la modalidad funciona mediante convocatorias que realizan las gobernaciones departamentales respectivas, a todas las organizaciones que reúnan los requisitos solicitados. Así mismo, al igual que convocan a organizaciones ponentes, también concitan a organizaciones acompañantes. La idea central en la iniciativa es el desarrollo de las comunidades a parir del trabajo participativo de sus miembros, el esfuerzo conjunto, la solidaridad y la cooperación. Esta modalidad en cierta forma elimina el despilfarro de recursos, la dispersión de esfuerzos.

Las Buenas Prácticas desde los inicios de la humanidad consciente (desde la separación de la horda de animales) ha sido una preocupación del hombre. En los orígenes de la humanidad los alimentos han sido producidos para satisfacer las necesidades biológicas sentidas por los individuos que su afán por el consumo de productos inicuos ha buscado diversas formas de producirlos y preservarlos. Hoy en día esta práctica se ha mejorado y de una técnica rudimentaria ha pasado a una aplicación tecnológica que se transfiere mediante procesos de capacitación, adiestramiento y formación.

Las Buenas Prácticas Agrícolas $\mathrm{BNP}^{3}{ }^{3}$ son las prácticas aplicadas en las unidades productivas desde la planeación del cultivo hasta la cosecha, el

3 En el documento Mis Buenas Prácticas Agrícolas "Guía para agro empresarios" (2007) Ministerio de Agricultura/Instituto Colombiano Agropecuario ICA (2009)- se puede encontrar un 
empaque y transporte del alimento (que pueden ser frutas, hortalizas y otros) con el propósito de asegurar la conservación de su buen estado y desde luego el estar apto para el consumo humano., en asocio directo con la conservación del medio ambiente y la seguridad y/o bienestar de los trabajadores del campo. En el mismo sentido y en el ámbito internacional el Grupo de Agricultura de la Oficina Regional de la FAO para América Latina y el Caribe (2007) viene desarrollando actividades de orientación, adiestramiento y socialización en las diferentes regiones del mundo con el firme propósito de difundir los conceptos básicos de las Buenas Prácticas Agrícolas (BPA), con el propósito de: orientar los sistemas de producción hacia una agricultura sostenible y ecológicamente segura, obtener productos inocuos y de mayor calidad, contribuir a la seguridad alimentaria a través de la generación de ingresos por acceso a mercados y mejorar las condiciones laborales de los productores y de sus familias (Colombia. Ministerio de Agricultura/Instituto Colombiano Agropecuario ICA, 2009, p.p. 1-17).

Esta labor ha necesitado de múltiples actividades cobijadas bajo la estrategia aunar esfuerzos para contribuir en la erradicación de la deficiencia alimentaria, la pobreza y la preservación de los alimentos, fomentando una cultura de sana productividad y manipulación, es decir Buenas Prácticas Agrícolas. El proceso y las acciones no se han quedado como una práctica en la agricultura sino que ha trascendido hacia las prácticas organizativas, las relaciones familiares y comunitarias, de tal manera que actualmente se hace relación a estas en el sentido de utilizar buenas prácticas que, combinas con las BPA, redunden de manera integral en el desarrollo armónico de los pueblos con la naturaleza y el medio ambiente.

En Colombia, la preocupación por la alta contaminación que esta sufriendo el planeta Tierra y en forma similar el impacto de las enfermedades que se transmiten por la ingesta de alimentos, ha disparado la atención de la sociedad y la ha dispuesto de tal modo que cobijada por la alerta en que se ha declarado el

referente pedagógico de lo que representa la transferencia de tecnología en el uso de buenas prácticas agrícolas. p.p. 1-17 
Vol. 6, No. 1 Diciembre de 2014 pp. 121 - 135

mundo, se han propuesto como objetivo "mejorar las condiciones y calidad con que se cultivan y producen los alimentos" (2005) situación que ha generado el surgimiento de nuevas propuestas que puestas al servicio campo contribuyen en mejorar, tal como lo plantea el Ministerio de Agricultura, tanto las condiciones de trabajo de los productores, como los productos que de allí salen a los mercados. Vendedores y consumidores se han dado cuenta que deben invertir en su salud; los primeros, sacando al mercado productos más limpios y sanos y, los segundos, consumiendo alimentos que hayan sido tratados con Buenas Prácticas. (2005)

En este sentido, hacer referencia de Buenas Prácticas Agrícolas o BPA es un reto, pero así mismo, responsabilidad y una gran oportunidad de negocio, porque permite a los pequeños productores entrar a mercados que tienen un mayor interés por el cuidado del medio ambiente y la salud humana. El interés actual se orienta por la transferencia de información para que en la práctica se implementen actividades con la aplicación de Buenas Prácticas Agrícolas y así los productores se enteren de lo que necesitan para que el negocio incluya el uso y manejo de estas. En la actualidad y desde la preocupación mundial por la calidad de los alimentos, la conservación de los mismos, la manipulación y la responsabilidad social que ello conlleva, se han adelantado la promulgación de normas jurídicas, técnicas, sociales, administrativas y comerciales relacionadas con la sanidad, el embalaje, la producción, la comercialización, el control de calidad, bodegaje, conservación, el transporte, etcétera que apuntan hacia la protección del consumidor final (también incluye al productor), representado por la sociedad global.

Tal escalada se ha materializado en un sinnúmero de eventos pedagógicos en los cuales se han vinculado profesionales de la educación, administradores, abogados, investigadores, ambientalistas, ingenieros (químicos, forestales, agrónomos) y otros que han volcado sus conocimientos y experiencias al servicio del mejoramiento de las condiciones de producción y demás procesos relacionados con los alimentos. Los eventos pedagógicos han permitido la construcción de metodologías, herramientas y estrategias de bajada de estos 
Vol. 6, No. 1 Diciembre de 2014 pp. $121-135$

conocimientos a los escenarios y a los actores, de acuerdo con las características de cada quien y la naturaleza de las ocupaciones, sus costumbres, estilos de vida, organizaciones, tratando en todo sentido, lograr el máximo de aprovechamiento de los recursos que se disponen. El mundo de la literatura está provisto de informes, boletines, manuales, cartillas, documentos, libros, ayudas didácticas (técnicas y tecnológicas) con las cuales se quiere que los actores tengan pleno conocimiento de los compromisos y retos que a cada quien compete con su participación.

En esta perspectiva la literatura y los apoyos didácticos que se han implementado es profusa y se ajusta a las necesidades de todo tipo de público. En ella se impreso un lenguaje sencillo, narrativo y explicativo que recrea los conocimientos y facilita la comprensión de explicaciones para la acción y la construcción de herramientas de acuerdo con la naturaleza de cada escenario y características de los actores.

\section{Los primeros hallazgos provisionales}

Como en la gran mayoría de las comunidades de la Costa Atlántica y aún en muchas regiones de Colombia los cultivos en las huertas se llevan a cabo en forma rudimentaria y artesanal, sujetos a las "buenas y malas épocas", con poca o ninguna disponibilidad de agua, solo aquella que provenía de la naturaleza. Es una constante amenaza para los proyectos que necesitan de agua, no solo en Colombia sino en algunas áreas de Latinoamérica como lo establecen Moreyra y col. (2012) en un interesante estudio. Aunque algunas zonas han mejorado con el sistema de regadío artificial, en otras se presenta escasez y aún tienen que padecer las inclemencias de los intensos veranos.

Independientemente de estas situaciones en el campo se mantenía un enfrentamiento entre cambiar la forma de pensar y establecer relaciones productivas distintas a las que han marcado parte de la trayectoria de su vida agropecuaria o permanecer con la visión que se había constituido en la única visión con la cual se enfrentaban a la vida cotidiana y en todas las relaciones construidas y por hacer. Este fue el escenario que se conocía en la comunidad de Santa Cruz, corregimiento de Luruaco, departamento del Atlántico, al norte de 
Vol. 6, No. 1 Diciembre de 2014 pp. 121 - 135

Colombia, al momento de irrumpir el Programa Alianzas Estratégicas, liderado por el Ministerio de Agricultura, la Gobernación de Atlántico y Prodesarrollo.

La comunidad a través de la Fundación Horizonte Verde presentó el proyecto "Alianza para la Siembra y Comercialización de 57,5 Hectáreas de Mango de Hilaza para Mejorar los Ingresos a los Fruticultores del Corregimiento de Santa Cruz en el Municipio de Luruaco", el cual fue aprobado no sin antes enseñar a los campesinos afiliados a la entidad los pormenores y los detalles que formaban parte de la organización, advirtiéndoles que el tiempo de aprobación del estudio con respecto a las primeras actividades de ejecución de actividades, sería lento y no estaría exento de dificultades.

La organización también debía cambiar. Una entidad que no estaba planteada para afrontar el desarrollo de proyectos productivos, sin ninguna experiencia en la siembra de mango, sin la práctica de comercialización debía afrontar el reto de establecer convenios con otras organizaciones y asumir la responsabilidad de nuevos y novedosos escenarios de la vida comercial.

Después de aprobado el proyecto, vinieron los primeros meses de un papeleo que debería registrarse para el cumplimiento de requisitos entre estos, pólizas de manejo, actas de reuniones, capacitaciones a los agricultores y el establecimiento de alianzas para el mercadeo de los productos.

Los detalles y la descripción de cada uno de los hallazgos encontrados en el estudio se detallan a continuación:

- Capacitación a los productores de la Fundación en siembra, cosecha, postcosecha y comercialización de mango. Los "sembradores de mango" tenían la costumbre arraigada de plantar el árbol y esperar buen tiempo para recolectar los primeros frutos. De ocho a diez años era la espera, afrontando las desventajas de la premura del tiempo, sobre todo si era de intenso verano. Con el cambio de las condiciones climáticas ya no existe la predicción del campesino de otrora del agricultor que conocía de antemano, cuando llovería. Las fuentes de agua han disminuido considerablemente dificultando aún más las actividades para 
Vol. 6, No. 1 Diciembre de 2014 pp. 121 - 135

la siembra de mango. Los responsables de la capacitación se refirieron a que en la comunidad no existían sembradores de mango sino, recolectores.

Las jornadas de capacitación se iniciaron con la metodología ECAS (Escuelas Escuela de Campos) expresada en la escogencia de una granja o el predio de cualquiera de los campesinos favorecidos (cercano a la comunidad) para facilitar el desarrollo de los talleres, los cuales consistieron en poner en escena los elementos con los cuales estaban familiarizados los campesinos. En el informe suministrado por la Gerencia en formación del proyecto se hace un recuento de las actividades de gestión, ejecutadas para este componente.

Convocatorias a la capacitación de los productores de mango beneficiarios del proyecto.

Esta actividad se lleva a cabo con la Asociación Hortícola y Frutícola de Colombia ASOHOFRUCOL de la cual la Fundación Horizonte Verde HORVE es miembro. La ejecutoria del componente capacitación se viene desarrollando desde el mes de febrero del presente año y a ella asisten en promedio 25 productores de mango entre beneficiarios del proyecto y otros interesados en el tema siembra y poda de mango. El escenario de los eventos lo constituye el corregimiento de Santa Cruz y la metodología que se emplea es la denominada Escuela de Campos ECAS. La actividad que despliega la gerencia en este aspecto consiste en la motivación hacia los productores para que asistan a la jornada y para ello se utiliza la estrategia de invitación personal casa por casa. Así mismo, la actividad sirve de manera simultánea como animadora de las expectativas por el proyecto y cohesionadora del grupo. El evento se lleva a cabo cada 15 días el día miércoles de cada mes. (Guillermo Reales, Gerente en formación, 2012)

Las actividades de capacitación culminaron en el mes de diciembre de ese mismo año y los productores experimentaron un cambio, no solo en las relaciones 
de producción, sino en todas sus actuaciones. El Gerente en Formación notifica informa sobre la nueva concepción:

\section{Culminación de la capacitación de los productores de mango beneficiarios del proyecto.}

En el mes de noviembre (a finales) culminaron las actividades de capacitación organizadas a través del convenio entre la Asociación Hortícola y Frutícola de Colombia ASOHOFRUCOL y la Fundación Horizonte Verde HORVE con un total de 32 productores capacitados, de los cuales 23 son beneficiarios del proyecto de la referencia. Esta actividad adscrita al componente capacitación como ya se había informado antes, había iniciado en el mes de febrero del presente año. Se acordó con los productores la entrega de constancia de asistencia a cada participante en la fecha del 27 de diciembre en el corregimiento de Santa Cruz - Luruaco, dejando encargado a la Gerencia del proyecto de los preparativos para la ceremonia.

Es importante hacer mención del buen balance que los productores hicieron de la actividad, pues la calificaron como generadora de conocimientos técnicos de los cuales no disponían con relación al producto que ocupará su atención en el proyecto. De igual manera es destacable el aspecto integrador de la actividad y el interés que despertó en los participantes, quienes se están preparando para los próximos compromisos de capacitación al interior de los componentes del proyecto. (2012)

- La organización Horizonte Verde. El inicio del cambio de actitud con respecto a las Buenas Prácticas Agrícolas. En la interpretación que del desarrollo han hecho Austin, Herrero \& Reficco (2007), la nueva ruta se muestra sobre las alianzas sociales estratégicas como una nueva opción de despegue con participación y responsabilidad. En un gesto que se caracterizó por la puesta en práctica de lo contrario a las recomendaciones de lo que significa para la organizaciones la aplicación de Buenas Prácticas Agrícolas, la organización Horizonte Verde asumió el reto de presentar una propuesta de desarrollo social y económico cuyo objetivo principal giraba en torno al mejoramiento de las condiciones de vida familiares de los productores de mango en el corregimiento de Santa Cruz - municipio de Luruaco, Departamento del Atlántico- mediante los 
ingresos producidos por la siembra y comercialización de 57.5 hectáreas de mago de hilaza. Se argumenta que la organización no estaba dentro de las estrategias para hacer Buenas Prácticas Agrícolas por cuanto ni siquiera hizo parte del grupo que tenía a su cargo la organización del proyecto; tampoco había contribuido en su elaboración, sino que el documento fue preparado por quienes fungían como acompañantes en la gestión.

Los potenciales beneficiarios tenían una doble connotación en su contra: no conocían en detalles (no participaron en la elaboración y/o formulación de la propuesta) los aspectos consignados en la propuesta; tampoco conocían a la organización que los representaría. Sin embargo, peso a tales desatinos, el proyecto fue aprobado y paso a la instancia en la cual se definiría la viabilidad del mismo, las utilidades, la permanencia. Los aspectos anteriormente descritos influyeron de manera notoria en la selección de las personas (los potenciales beneficiarios inscritos) y se constituyeron en una amenaza para la sostenibilidad de la propuesta.

La nueva mentalidad y cambio de actitud se expresa a partir de los compromisos que la organización debe asumir, entre ellos las reuniones formales con los productores de mango afiliados al proyecto, en búsqueda de la estabilización de una base de beneficiarios, cuyos resultados en Gerente en formación detalla de la siguiente manera:

En estos encuentros se les recuerda el compromiso que cada uno de los beneficiarios ha contraído con la ejecución del proyecto. En ellos se desarrollan también actividades de información relacionadas con la organización y la responsabilidad que cada productor tiene con su fortalecimiento tanto en lo económico como en lo logístico. Las reuniones han servido también para re-afirmar la voluntad de permanencia de los actuales beneficiarios, las solicitudes de nuevos ingresos a la organización y la ratificación por escrito de quienes habían anunciado su decisión de no seguir con el proyecto, al no reunir las condiciones establecidas para la 
Vol. 6, No. 1 Diciembre de 2014 pp. 121 - 135

vinculación. A la fecha se encuentra totalmente definida la base de productores beneficiados con el proyecto de alianza. (2012)

Otro aspecto de suma importancia que muestra la nueva actitud de la organización hace referencia a la Gestión de contrapartidas que corresponden a las entidades comprometidas con el proyecto. El Gerente en Formación hace un balance de estas actividades propias de la organización:

Con el acompañamiento de la OGA se ha venido trabajando en la definición de las contrapartidas correspondientes al Fondo de Adaptación y la Gobernación del Departamento del Atlántico, es así como a la fecha estamos enviando la carta suscrita por el representante de la Fundación, solicitando la apertura de cuenta con cargo al Fideicomiso Fiducoldex Horve para lo referente al depósito de los recursos del Fondo.

Para la contrapartida de la Gobernación venimos acopiando la información requerida por la Secretaría de Desarrollo Económico del Departamento. Se aspira estar radicando tanto los formatos como documentación de la organización antes de culminar el presente mes de noviembre. (2012)

Finalmente, otro de los aspectos que llama poderosamente la atención en el cambio de mentalidad de la organización, está relacionada con lo que la entidad anuncia como un slogan: Compromiso con el planeta. Este compromiso lo asume más que la aplicación de buenas prácticas agrícolas, como un gesto de responsabilidad social organizacional, desde la gestión en los componentes definidos para el proyecto. Con la vinculación de los profesionales que estarán apoyando las actividades de aprestamiento, asesoría y acompañamiento a los productores beneficiarios se ha dinamizado una gran actividad en cada uno de ellos y la gestión desplegada se clasifica en tres aspectos: 
- Componente agroindustrial. Con la vinculación del Ingeniero Agrónomo Hernán Laguna fueron visitadas durante el mes de Noviembre y parte del mes de Diciembre, cada una de las 23 parcelas destinadas por los productores beneficiarios para el proyecto siembra de mango (ver informe de la actividad). En ellas fueron tomadas muestras de suelo y cada productor definió, de acuerdo con la asesoría del Ingeniero, el sitio donde se establecerían las plántulas de mango, antes de sembrarlas en el lugar definitivo. En cada parcela el productor firmó el registro de la respectiva visita, validando el apoyo técnico que estaba recibiendo por parte del profesional. Esta actividad se desarrolló durante una semana y todos los productores fueron atendidos en sus solicitudes.

- Componente empresarial. Se realizó el acompañamiento al profesional encargado de este componente, el Administrador de Empresas Jairo Aguirre, quien de manera individual le suministraba indicaciones al productor y le inducía hacia los compromisos que adquiriría desde el punto de vista comercial con la organización y las demás entidades vinculadas al proyecto (ver informe de la actividad). El profesional explicó a los productores los requisitos y los documentos soportes que acompañarían el respaldo de la obligación. Se realizaría una reunión colectiva para mediados del mes de diciembre de 2012 con todos los productores, en la cual estarían firmando el pagaré como respaldo a la deuda del Fondo. Esta gestión se había adelantado antes con el Ministerio de Agricultura en el marco de la Estrategia Celuagronet.

- Componente ambiental. La gestión en este componente fue de acompañamiento permanente a la profesional encargada de las actividades de visitas de observación y asesoría a los productores. La Técnica Profesional Nazly Altamiranda es la encargada de suministrar explicaciones y hacer observaciones en cada una de las parcelas de los productores beneficiarios, relacionadas con el impacto que produce al medio ambiente la ubicación de un objeto en este $u$ otro lugar, como en el caso de las plántulas de mango, en el sitio provisional y luego el definitivo (ver informe de la actividad). Esta actividad se realizó durante una 
Vol. 6, No. 1 Diciembre de 2014 pp. $121-135$

semana y durante estas visitas los productores aprovecharon la oportunidad para conocer más detalles sobre los aspectos ambientales.

Los tres profesionales hicieron una charla colectiva sobre cada componente, de acuerdo con el compromiso que cada quien tiene en el proyecto y se comprometieron en el suministro de una Agenda de Trabajo para que cada productor programe su tiempo y pueda cumplir con las actividades del proyecto sin descuidar los otros compromisos de su cotidianidad.

\section{CONCLUSIONES}

La apreciación que deja la aplicación de Buenas Prácticas Agrícolas supone un reto en el cambio de actitud tanto de los beneficiarios del Proyecto no solo para la organización que lo lidera, en este caso la Fundación Horizonte Verde, sino para las personas adscritas al mismo y sus familias. Y recordar que la aplicación de Buenas Prácticas Agrícolas como lo establece José Ignacio Cadahía, va más allá de un "manejo integrado de los cultivos y unas Agrícolas entendidas como aquellas que contribuyen a las sostenibilidad de los sistemas agrícolas productivos y su entorno". (2012). No solo se trata del compromiso que asume la entidad consigo misma sino como la comunidad, con la sociedad del planeta. Tampoco es el compromiso que asume individualmente el productor, sino el trabajo en equipo que debe redundar en fortaleza para la organización y bienestar para la comunidad.

En cada uno de los eventos que desarrollaron los profesionales contratados para componente dejaron sus impresiones, las mismas que han servido para establecer que en algo se ha cambiado tanto en la persona como en la organización. Este cambio debe trasladarse a la familia.

\section{BIBLIOGRAFIA}


Austin, J., Herrero, G. \& Reficco, E. (2007). La Nueva Ruta: Alianzas sociales estratégicas. En: Harvard Bussines Review. Edición Especial (Agosto, 2007).

Colombia. Ministerio de Agricultura/Instituto Colombiano Agropecuario ICA (2009). Mis Buenas Prácticas Agrícolas "Guía para agro empresarios

Colombia. Ministerio de Agricultura. (2005). Buenas Prácticas Agrícolas. Bogotá: Ministerio de Agricultura y Desarrollo Rural.

España. AEPLA. (2012). Buenas Prácticas Agrícolas. En Horticultura -----. (2000). Las Buenas Práctica Agrícolas. En: Horticultura, mayo de 2000.

Fals Borda. O. (1998). Metodología de la IAP. Medellín.

Molano Camargo, F. (2012). Las organizaciones campesinas como escenarios de resistencia, creación de conocimiento y memoria. Bogotá: Universidad Distrital de Bogotá.

Moreyra, A. et. al. (2012). El acceso al agua de los agricultores familiares de la región pampeana: un análisis multidimensional. En: Mundo Agrario, vol. 12, no 24, primer semestre de 2012. 